\title{
Penning-trap mass spectrometry and mean-field study of nuclear shape coexistence in the neutron-deficient lead region
}

\author{
V. Manea, ${ }^{1,2,{ }^{*}}$ P. Ascher, ${ }^{2, \dagger}$ D. Atanasov, ${ }^{2}$ A. E. Barzakh, ${ }^{3}$ D. Beck, ${ }^{4}$ K. Blaum, ${ }^{2}$ Ch. Borgmann, ${ }^{2}$ M. Breitenfeldt,${ }^{5}$ \\ R. B. Cakirli, ${ }^{2, \dagger}$ T. E. Cocolios, ${ }^{6,}{ }^{\S}$ T. Day Goodacre,,${ }^{6,7}$ D. V. Fedorov, ${ }^{3}$ V. N. Fedosseev, ${ }^{7}$ S. George, ${ }^{2}$ F. Herfurth, ${ }^{4}$ \\ M. Kowalska, ${ }^{8}$ S. Kreim, ${ }^{2,8}$ Yu. A. Litvinov, ${ }^{4}$ D. Lunney, ${ }^{1}$ B. Marsh, ${ }^{7}$ D. Neidherr, ${ }^{4}$ M. Rosenbusch, ${ }^{9}$ R. E. Rossel, ${ }^{7,10}$ \\ S. Rothe, ${ }^{7,10}$ L. Schweikhard, ${ }^{9}$ F. Wienholtz, ${ }^{9}$ R. N. Wolf, ${ }^{9,}{ }^{\|}$and K. Zuber ${ }^{11}$ \\ ${ }^{1}$ CSNSM-IN2P3-CNRS, Université Paris-Sud, 91406 Orsay, France \\ ${ }^{2}$ Max-Planck-Institut für Kernphysik, 69117 Heidelberg, Germany \\ ${ }^{3}$ Petersburg Nuclear Physics Institute, NRC Kurchatov Institute, 188300, Gatchina, Russia \\ ${ }^{4}$ GSI Helmholtzzentrum für Schwerionenforschung GmbH, 64291 Darmstadt, Germany \\ ${ }^{5}$ Instituut voor Kern- en Stralingsfysica, 3001 Heverlee, Belgium \\ ${ }^{6}$ School of Physics and Astronomy, University of Manchester, Manchester M13 9PL, United Kingdom \\ ${ }^{7}$ CERN, Engineering Department, CH-1211 Geneva, Switzerland \\ ${ }^{8} \mathrm{CERN}, \mathrm{CH}-1211$ Geneva, Switzerland \\ ${ }^{9}$ Ernst-Moritz-Arndt-Universität, Institut für Physik, 17487 Greifswald, Germany \\ ${ }^{10}$ Institut für Physik, Johannes Gutenberg Universität, 55099 Mainz, Germany \\ ${ }^{11}$ Technische Universität Dresden, 01069 Dresden, Germany
}

(Received 7 September 2016; revised manuscript received 3 March 2017; published 24 May 2017)

\begin{abstract}
We present a study of nuclear shape coexistence in the region of neutron-deficient lead isotopes. The midshell gold isotopes ${ }^{180,185,188,190} \mathrm{Au}(Z=79)$, the two long-lived nuclear states in ${ }^{197} \mathrm{At}(Z=85)$, and the neutron-rich nuclide ${ }^{219}$ At were produced by the ISOLDE facility at CERN and their masses were determined with the high-precision Penning-trap mass spectrometer ISOLTRAP. The studied gold isotopes address the trend of binding energies in a region of the nuclear chart where the nuclear charge radii show pronounced discontinuities. Significant deviations from the atomic-mass evaluation were found for ${ }^{188,190} \mathrm{Au}$. The new trend of two-neutron separation energies is smoother, although it does reveal the onset of deformation. The origin of this effect is interpreted in connection to the odd-even staggering of binding energies, as well as theoretically by HartreeFock-Bogoliubov calculations including quasiparticle blocking. The role of blocking for reproducing the large odd-even staggering of charge radii in the mercury isotopic chain is illustrated.
\end{abstract}

DOI: 10.1103/PhysRevC.95.054322

\section{INTRODUCTION}

The theoretical description of nuclear structure is made difficult not only by uncertainties in the modeling of the nucleon-nucleon interaction but also by the sheer number of constituents of the atomic nucleus, making the problem too difficult to solve. Efforts are ongoing to extend $a b$ initio

\footnotetext{
*Present address: CERN, CH-1211 Geneva, Switzerland; vladimir.manea@cern.ch

†Present address: Centre d'Etudes Nucléaires de BordeauxGradignan, 19 Chemin du Solarium, CS 10120, F-33175 Gradignan, France.

${ }^{\ddagger}$ Present address: Istanbul University, Department of Physics, 34134, Istanbul, Turkey.

${ }^{\S}$ Present address: Instituut voor Kern- en Stralingsfysica, 3001 Heverlee, Belgium.

"Present address: ARC Centre of Excellence for Engineered Quantum Systems, School of Physics, University of Sydney, NSW 2006, Australia.
}

Published by the American Physical Society under the terms of the Creative Commons Attribution 3.0 License. Further distribution of this work must maintain attribution to the author(s) and the published article's title, journal citation, and DOI. approaches to mid-mass nuclei [1-3], many by modeling the nuclear Hamiltonian using chiral effective field theory and renormalization-group methods [4].

Most nuclei are, however, still treated with drastic approximations to the nuclear many-body problem or restrictions thereof, the interactions being phenomenologically adjusted to nuclear data. The nuclear shell model [5], for example, is still restricted with respect to the accessible valence spaces and each of them requires its own adjustment of the nuclear Hamiltonian. For the more global approaches, recent studies on the uncertainties of density-functional-type models [6] show that the parameters of the nuclear energy functional are insufficiently constrained, leading to questionable predictive power [7]. This in turn casts doubt on simulations of astrophysical scenarios such as the r-process, which largely rely on theory to provide the many required nuclear properties (see Ref. [8] for a recent study).

Recent works in the $a b$ initio framework emphasize the distinction between actual observables and model concepts in nuclear-structure theory, in connection to the presumed shell structure [9] and even the nuclear Hamiltonian itself [10]. Nevertheless, while efforts to build a microscopic manybody nuclear theory continue, phenomenological approaches remain an important tool to classify the manifestations of nuclear structure with the variation of proton and neutron 
number and to highlight regions of interest for experiment. In this context, it is still interesting to confront their predictions with experiment and check the internal consistency of their theoretical constructs such as nuclear shells and nuclear deformation outside their original domain of parameter adjustment.

Despite their lack of spectroscopic accuracy, mean-field or density-functional-type approaches [11] remain an interesting tool to track the changes in structure along isotopic or isotonic chains: One often encounters in midshell regions sudden changes in the trends of nucleon separation energies or mean-square charge radii, accompanied by a change of the spacing of the yrast level sequence towards the pattern of a quantum-mechanical rigid rotor [12]. In the mean-field framework, it is in these regions that the intrinsic nuclear shape changes from almost spherical to well deformed, which produces effects on the computed nuclear properties that can be observed experimentally. Characteristic of the nuclear mean field is the existence of more than one local energy minimum with respect to variations of the nuclear wave function. The fingerprints of these equilibrium solutions are present in the excitation patterns of nuclei. A remarkable example is that of ${ }^{186} \mathrm{~Pb}$ [13], which has three $0^{+}$states "coexisting" at low excitation energy, corresponding to different mean-field configurations. With the variation of proton and neutron number, the ordering in energy of such coexisting states can change, usually accompanied by major changes in the trends of ground-state properties. Consequently, predicting the trend of observables in the mean-field approximation requires the correct descriptions not only of the deformation of every individual equilibrium configuration but also of their energy ordering.

In the present work, we report about a study of nuclear structure in the neutron-deficient lead region, which is well known for its variety of phenomena related to shape coexistence [14]. Studies performed over the past three decades by different methods have revealed a rapid evolution of nuclear structure with proton number. This is vividly illustrated by the nuclear charge radii in the region. Their trend along the lead isotopic chain $(Z=82)$ has a smooth evolution, expected for a magic proton number [15], but in the gold chain, with only three protons fewer $(Z=79)$, it shows a significant increase at neutron number $N=107$ [16-19]. In between, the thallium ground states $(Z=81)$ resemble more the behavior of the neighboring lead isotopes (see also the recent discussion [20]), while the long-lived thallium isomers have significantly larger charge radii [21]. The mercury isotopes $(Z=80)$ are the first to exhibit an increase in the ground-state charge radii at $N=105$ [22,23], but a strong odd-even staggering ensues $[24,25]$, suggesting the proximity in energy of very different nuclear states, which alternate with neutron number. This proximity is also apparent from the spectroscopy of even-even mercury isotopes [26], but it is still not obvious why the odd isotopes have a reverse ordering in energy. According to the 2012 atomic-mass evaluation (AME) [27], the two-neutron separation energies along the gold and mercury isotopic chains seem to be less sensitive to these phenomena. This behavior is at odds with the neutron-rich isotopes of mass $A \approx 100$, which at neutron number $N=60$ exhibit a concomitant increase in charge radii and two-neutron separation energies (see, for example, the multiobservable comparison of Ref. [12]).

In this work, we present new mass measurements of gold isotopes in the region of the discontinuity in the trend of charge radii, performed at ISOLDE/CERN with the Penning-trap mass spectrometer ISOLTRAP [28,29]. These investigations are part of a larger program of mass measurements in the gold and astatine isotopic chains and the masses of two astatine isotopes are also presented here. The new data help us refine the knowledge of binding energies along the gold isotopic chain and investigate a discontinuity in two-neutron separation energies at neutron number 109 . To interpret the data in the region, including the new experimental findings, we perform mean-field calculations, which, as discussed above, naturally accommodate nuclear-structure concepts such as nuclear deformation, shape coexistence, and shape transition.

The article is organized as follows. The first section describes the ISOLTRAP setup and the techniques used for the mass determination. The second section details the beam times during which the data were measured, as well as the measurement and the analysis procedures. The third section presents new Hartree-Fock-Bogoliubov (HFB) calculations and discusses in this framework the structure of nuclei in the neutron-deficient lead region.

\section{EXPERIMENT}

The nuclides studied in this work were produced by the ISOLDE radioactive ion-beam facility at CERN [30] by proton-induced reactions on a thick uranium carbide $\left(\mathrm{UC}_{\mathrm{x}}\right)$ target. The protons were delivered by CERN's protonsynchrotron booster (PSB) in pulses accelerated to $1.4 \mathrm{GeV}$ kinetic energy, providing an average current of up to $2 \mu \mathrm{A}$. The target was heated to ca. $2000^{\circ} \mathrm{C}$, which thus allowed releasing the reaction products by thermal diffusion and effusion. Some of the unwanted products were ionized on the walls of the heated transfer line between the target and the ISOLDE beam-transport system. The gold and astatine isotopes were selectively ionized by the resonance-ionization laser ion source (RILIS) of ISOLDE [31] using a three-step ionization scheme. The mixed ion beams (nuclides of interest and surface-ionized contaminants) were then accelerated to a kinetic energy of 30-50 keV and transported through the ISOLDE magnetic separators, which have sufficient resolving power to select the species of a certain atomic mass number.

The ISOLTRAP setup, schematically presented in Fig. 1, currently consists of four ion traps (described in more detail in Ref. [28,32]). The first is a linear, segmented, radio-frequencyquadrupole (RFQ) cooler and buncher, where the beam from ISOLDE is accumulated for a certain period of time, cooled by collisions with helium buffer gas and then ejected as a short ion pulse. A pulsed drift tube is used to reduce the energy of the beam from the ISOLDE transport energy to $\approx 3.2 \mathrm{keV}$. The ion bunch is then captured in the multireflection timeof-flight mass spectrometer (MR-ToF MS) [32,33], where, following multiple reflections between electrostatic mirrors, it is separated in its different charge-over-mass components. They can be either observed with an ion detector placed behind the MR-ToF MS or separated from contaminants using a 


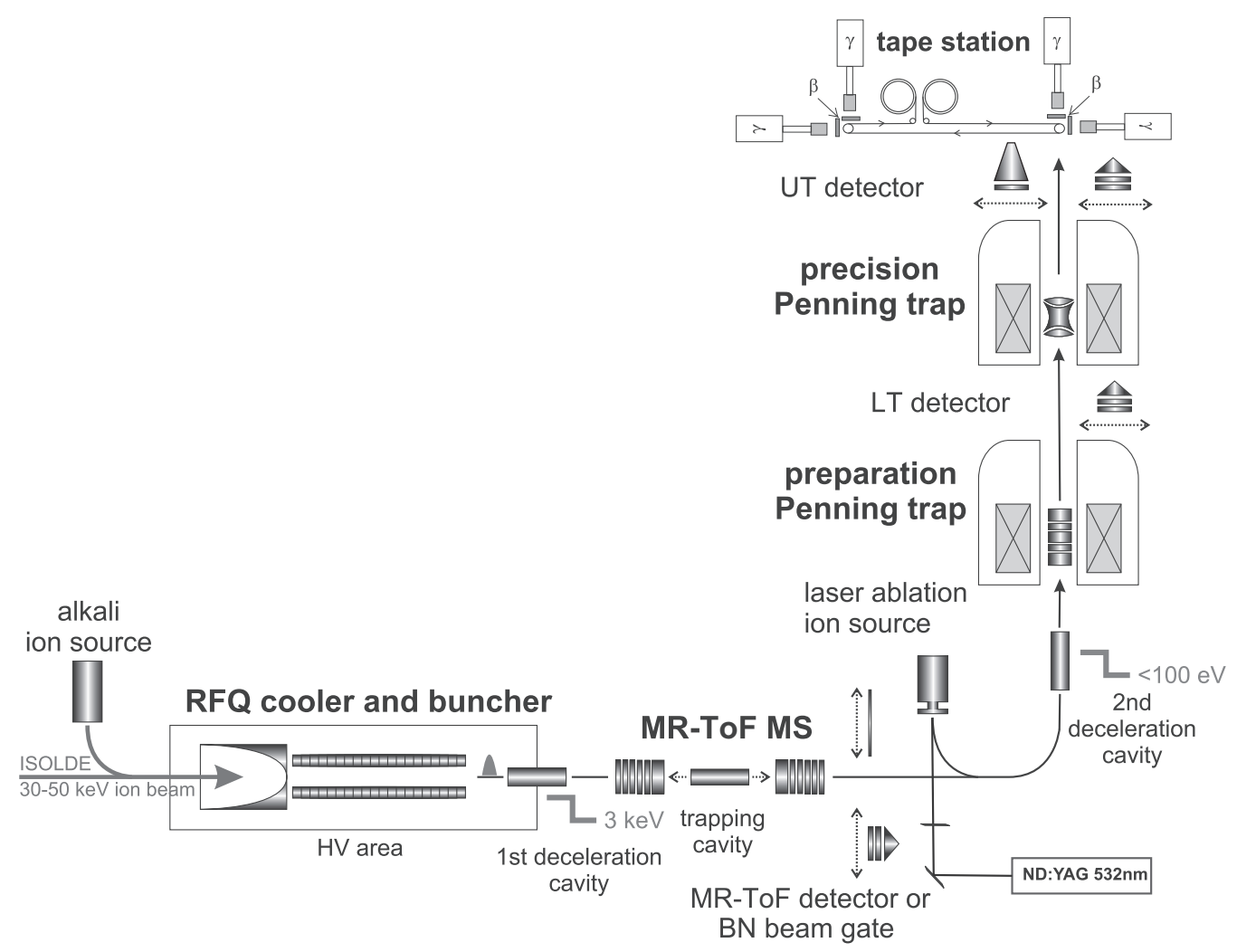

FIG. 1. Schematic view of the ISOLTRAP experiment. For details, see text.

Bradbury-Nielsen beam gate (BN beam gate) [34]. A mass resolving power above $10^{5}$ can be achieved within only a few tens of milliseconds trapping time in the MR-ToF MS [35]. The purified ion bunch then undergoes a $90^{\circ}$ bend and enters the vertical transport section of ISOLTRAP, which contains two Penning traps. The first is a cylindrical trap (preparation Penning trap [36]), used to cool and purify the ion bunch by mass-selective resonant buffer-gas cooling [37]. To reduce the ions' kinetic energy to less than $100 \mathrm{eV}$, enough for capturing them in the preparation Penning trap, a second pulsed drift tube is used. Following the purification and cooling in the preparation trap, the ion bunch is transported to the precision Penning trap, where the cyclotron frequency of the ion of interest is determined using the time-of-flight ion-cyclotron-resonance (ToF-ICR) technique [38].

The cyclotron frequency $v_{c, x}$ of the ion of interest $X$ is related to its mass $m_{\text {ion, } x}$ by the relationship $v_{c, x}=$ $q B /\left(2 \pi m_{\text {ion, } x}\right)$, where $q$ is the ion charge (equal to the elementary charge in our case, given that singly charged ions are investigated) and $B$ is the magnetic-field induction. Since it is difficult to measure the magnetic field with enough precision, one also measures the cyclotron frequency $v_{c \text {,ref }}$ of a reference ion of known mass and from it the frequency ratio:

$$
r_{\mathrm{ref}, x}=\frac{v_{c, \mathrm{ref}}}{v_{c, x}}=\frac{m_{\mathrm{ion}, x}}{m_{\mathrm{ion}, \mathrm{ref}}} .
$$

From the measured frequency ratio, one obtains the atomic mass of the species of interest:

$$
m_{x}=r_{\mathrm{ref}, x}\left(m_{\mathrm{ref}}-m_{e}\right)+m_{e},
$$

where $m_{\text {ref }}$ is the atomic mass of the reference species and $m_{e}$ is the electron mass.

The gold and astatine isotopes were studied in two different experiments, as summarized in Table I, where the total storage times in the MR-ToF MS and preparation trap are also presented. The trapping time in the MR-ToF MS is determined by the desired number of ion laps, which explains why the values in the corresponding column are different for ions of different mass. All species were studied as singly charged ions and in all cases the reference ion was ${ }^{133} \mathrm{Cs}^{+}$from an off-line ion source. In the first experiment, the mass of ${ }^{185} \mathrm{Au}^{+}$was measured, while the second, which itself was split in two parts due to the requirement of different laser ionization schemes, addressed the masses of ${ }^{197 g, m, 219} \mathrm{At}^{+}$and ${ }^{180,188,190} \mathrm{Au}^{+}$. The number of ToF-ICR measurements and the corresponding measurement time for each of them is shown in the last column of Table I.

The schemes employed for the laser ionization of the gold and astatine isotopes are recent developments at ISOLDE [41,42], with the latter marking also a first determination of the ionization potential of astatine. The laser ionization of the gold isotopes was performed using broad-band lasers of 10-20 GHz linewidth, while for the astatine isotopes a narrow-band laser system was used for one of the excitation steps, having a linewidth on the order of $\approx 1 \mathrm{GHz}$ [43]. The narrow-band arrangement allowed having sensitivity to different hyperfine-structure components, thus offering the possibility to enhance the purity of isomeric beams by tuning the laser frequency to a value which favors the ionization of only one nuclear state. RILIS as a tool for isomeric 
TABLE I. Parameters describing the production, preparation, and measurement of the gold and astatine nuclides discussed in this work. The total trapping times for the MR-ToF MS and preparation trap are rounded and displayed in the corresponding columns. The last column presents the number of ToF-ICR measurements and exact excitation time in the precision trap. The superscript $R$ indicates that a Ramsey-type resonance was recorded $[39,40]$ (the times reflect the used excitation pattern). For details, see text.

\begin{tabular}{|c|c|c|c|c|c|c|c|c|}
\hline \multicolumn{5}{|c|}{ Production } & \multicolumn{4}{|c|}{ Preparation/measurement } \\
\hline May 2012 & $\mathrm{UC}_{\mathrm{x}} / \mathrm{Ta}$ & RILIS & $30 \mathrm{keV}$ & GPS & ${ }^{185} \mathrm{Au}^{+}$ & $32.7 \mathrm{~ms}$ & $360 \mathrm{~ms}$ & $\begin{array}{l}1 \times 1.2 \mathrm{~s} \\
3 \times 3 \mathrm{~s} \\
1 \times 5 \mathrm{~s}\end{array}$ \\
\hline \multirow[t]{4}{*}{ Oct 2012} & $\mathrm{UC}_{\mathrm{x}} / \mathrm{Ta}$ & RILIS & $50 \mathrm{keV}$ & HRS & ${ }^{197} \mathrm{At}^{+}\left(I^{\pi}=9 / 2^{-}\right)$ & $34.1 \mathrm{~ms}$ & $210 \mathrm{~ms}$ & $\begin{array}{l}1 \times 100 \mathrm{~ms} \\
2 \times 30-240-30 \mathrm{~ms}^{R}\end{array}$ \\
\hline & & & & & ${ }^{219} \mathrm{At}^{+}$ & $35.6 \mathrm{~ms}$ & $110 \mathrm{~ms}$ & $2 \times 1.2 \mathrm{~s}$ \\
\hline & & & & & ${ }^{180} \mathrm{Au}^{+}$ & $32.2 \mathrm{~ms}$ & $110 \mathrm{~ms}$ & $\begin{array}{l}1 \times 1.2 \mathrm{~s} \\
1 \times 1.8 \mathrm{~s} \\
1 \times 2.5 \mathrm{~s}\end{array}$ \\
\hline & & & & & ${ }^{188} \mathrm{Au}^{+}$ & $65.9 \mathrm{~ms}$ & $110 \mathrm{~ms}$ & $\begin{array}{l}2 \times 1.2 \mathrm{~s} \\
1 \times 3 \mathrm{~s}\end{array}$ \\
\hline
\end{tabular}

purification has been successfully exploited for a number of years at ISOLDE [44]. In addition to its typical use as an element-selective ion source, the RILIS is regularly applied for in-source laser spectroscopy for hyperfine-structure (HFS) and isotope-shift measurements [45]. In the following, some relevant details of each measurement will be addressed. The data analysis procedure follows closely the principles detailed in Ref. [46]. A summary of the experimental results obtained in this work are presented in Table II. The main sources for the mass values in the atomic-mass evaluation are cited in the last column of the table.

\section{A. ${ }^{190} \mathrm{Au}$}

The ${ }^{190} \mathrm{Au}$ beam was contaminated by the thallium isobar, but well separated using the MR-ToF MS, as shown in Fig. 2. The $I^{\pi}=11^{-}, 125-\mathrm{ms}$ half-life isomer was identified in this isotope in Ref. [47]. The frequency of the first step of the RILIS ionization scheme (the $6 s^{2} S_{1 / 2} \rightarrow 6 p^{2} P_{1 / 2}$ transition at $267.594 \mathrm{~nm}$ ) was thus scanned, in search for a distinct frequency which preferentially enhances the ionization of the isomer. The ion rate was recorded using the timeof-flight detector behind the MR-ToF MS, as described in Refs. [32,45]. No distinct ionization peak was observed in the wave-number range 12451.6-12454.6 $\mathrm{cm}^{-1}$ (values before frequency tripling), making the separation and study of the short-lived isomer impossible. A pure ${ }^{190} \mathrm{Au}^{+}$beam was subsequently transported to the precision Penning trap, where 1.2-s ToF-ICR measurements were performed. The long excitation time in the precision Penning trap ensured that, whatever amount of isomeric state might have been present in the beam, it would have decayed in the two Penning traps. A significant deviation to the mass value in the atomicmass evaluation was found [27], the ISOLTRAP mass being approximately $3 \sigma$ heavier than the evaluated one (considering the uncertainties of both values). The AME 2012 mass value is constrained both by a storage-ring measurement [48] and $\beta$-decay $Q$-value links to ${ }^{190} \mathrm{Pt}[49,50]$ and ${ }^{190} \mathrm{Hg}[50]$.

TABLE II. Measured frequency ratios with respect to ${ }^{133} \mathrm{Cs}^{+}$and derived mass excess $\left(M_{\mathrm{exc}}\right)$ values of the gold isotopes measured in this work. The half-lives $\left(T_{1 / 2}\right)$ of the isotopes from NUBASE 2012 [59] and the mass excess values from AME 2012 [27] are also given. For computing the mass excess, the ${ }^{133} \mathrm{Cs}$ mass value from the AME 2012 is used, namely 132.905451961(9) $u$, and the atomic mass unit value $u=931494.0023(7) \mathrm{keV} / c^{2}$. The last column specifies the main references contributing to the AME 2012 value. For references to $\alpha$ - and $\beta$-decay $Q$-value links which agree within the statistical uncertainty, only the earliest is cited.

\begin{tabular}{|c|c|c|c|c|c|c|}
\hline Isotope & $T_{1 / 2}$ & $r=v_{c, \text { ref }} / v_{c}$ & $\begin{array}{l}M_{\text {exc,ISOLTRAP }} \\
\left(\mathrm{keV} / c^{2}\right)\end{array}$ & $\begin{array}{c}M_{\text {exc,AME2012 }} \\
\left(\mathrm{keV} / c^{2}\right)\end{array}$ & $\begin{array}{c}\Delta_{\mathrm{TRAP}-\mathrm{AME}} \\
\left(\mathrm{keV} / c^{2}\right)\end{array}$ & References \\
\hline${ }^{180} \mathrm{Au}$ & $8.1(3) \mathrm{s}$ & $1.3541407041(400)$ & $-25627.25(4.95)$ & $-25594(20)$ & $-33.3(20.6)$ & {$[48,57]$} \\
\hline${ }^{188} \mathrm{Au}$ & $8.84(6) \mathrm{min}$ & $1.4142796434(220)$ & $-32371.33(2.72)$ & $-32277(15)$ & $-94.3(15.2)$ & {$[48,51,52]$} \\
\hline${ }^{190} \mathrm{Au}$ & $42.8(1.0) \mathrm{min}$ & $1.4293242637(281)$ & $-32833.53(3.48)$ & $-32883(16)$ & 49.5(16.4) & [48-50] \\
\hline${ }^{197} \operatorname{At}\left(I^{\pi}=9 / 2^{-}\right)$ & $388.2(5.6) \mathrm{ms}$ & $1.482207250(148)$ & $-6371.5(18.3)$ & $-6340(50)$ & $-31.5(53.2)$ & [63] \\
\hline
\end{tabular}

${ }^{\mathrm{a}}$ Excitation energy 45.6(22.8) keV. 


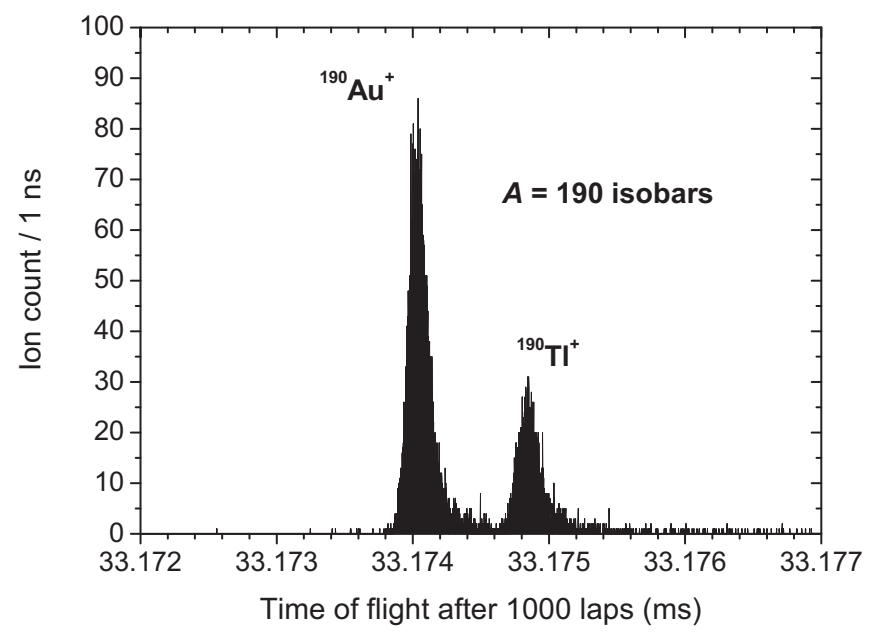

FIG. 2. Time-of-flight spectrum of $A=190$ isobars after 1000 laps in the MR-ToF MS, with the RILIS optimized for maximum Au ionization efficiency.

\section{B. ${ }^{188} \mathrm{Au}$}

A similar laser-ionization study as for ${ }^{190} \mathrm{Au}$ was performed for ${ }^{188} \mathrm{Au}$, in the search for a high-spin isomeric state, but no frequency was found for the selective ionization of an isomer in the wave-number range $12453.1-12454.6 \mathrm{~cm}^{-1}$. A pure ${ }^{188} \mathrm{Au}^{+}$beam was transported to the precision Penning trap, where the long storage time inhibits the subsequent detection of any 100-ms isomeric state that may have been present. A ToF-ICR measurement with 3-s excitation time was applied, but also did not reveal any long-lived isomer at low excitation energy. As for the case of ${ }^{190} \mathrm{Au}$, a significant deviation to the AME 2012 mass value was found, the ISOLTRAP value being 6 combined $\sigma$ lighter. As in the case of ${ }^{190} \mathrm{Au}$, the AME 2012 mass value is constrained both by storage-ring measurements [48,51] and $\beta$-decay $Q$-value links to ${ }^{188} \mathrm{Pt}$ and ${ }^{188} \mathrm{~Pb}$ [52].

$$
\text { C. }{ }^{185} \mathrm{Au}
$$

The beam of ${ }^{185} \mathrm{Au}^{+}$was significantly more abundant than the thallium contamination, given the low production cross section of ${ }^{185} \mathrm{Tl}$. Two long-lived nuclear states were proposed for ${ }^{185} \mathrm{Au}$ in Ref. [53] and confirmed in Ref. [54]. They have spins $I^{\pi}=1 / 2^{+}$and $5 / 2^{-}$, with half-lives on the order of minutes but unknown difference in energy. The ordering in energy between the two states is also not known. The beam of ${ }^{185} \mathrm{Au}^{+}$was transported to the precision trap, where several measurements at long excitation times were performed (up to $5 \mathrm{~s}$ ), with the purpose of increasing resolving power. The measurements revealed no presence of a second state. The conclusion is that either the two nuclear states are too close in energy to be resolved, or the production ratio differs significantly (by more than one order of magnitude). The measurement of maximum resolving power would have allowed completely separating two states differing in energy by $150 \mathrm{keV}$ or more. The single mass value extracted is one order of magnitude more precise than the AME 2012 value, but still lies within 1 standard deviation from it (combining the uncertainties of both values). The AME 2012 value is constrained by storage-ring measurements [48,51], by a $\beta$-decay $Q$-value link to ${ }^{185} \mathrm{Pt}$ [55], and by an $\alpha$-decay $Q$-value link to ${ }^{181} \operatorname{Ir}$ [56]. Considering the work of Ref. [17], the spin of the state produced and measured at ISOLDE is likely 5/2.

\section{D. ${ }^{180} \mathrm{Au}$}

The ${ }^{180} \mathrm{Au}^{+}$was significantly more abundant than any isobaric contamination. A scan of the frequency of the first RILIS excitation step was performed using the MR-ToF MS: A wide range around the central ionization peaks (12451.2-12455.0 $\mathrm{cm}^{-1}$ ) did not reveal the presence of any additional resonance indicative of a long-lived isomer. The pure ${ }^{180} \mathrm{Au}^{+}$beam was transported to the precision Penning trap, where three ToF-ICR measurements of increasing excitation time were performed, revealing a single nuclear state. The ISOLTRAP mass value deviates from the one in AME 2012 by approximately one combined standard deviation, but is 4 times more precise. The AME 2012 value is constrained both by a storage-ring measurement [48] and by alpha-decay $Q$-value links to ${ }^{176} \mathrm{Ir}[57]$ and ${ }^{184} \mathrm{Tl}$ [58].

$$
\text { E. }{ }^{197} \mathrm{At}
$$

The beam of ${ }^{197} \mathrm{At}^{+}$was ionized by the RILIS in narrowband operation mode. The beam was primarily contaminated by isobaric thallium and tantalum monoxide, which were eliminated using the MR-ToF MS. ${ }^{197}$ At contains two longlived nuclear states, one of $I^{\pi}=1 / 2^{+}$and half-life $2 \mathrm{~s}$ and another of $I^{\pi}=9 / 2^{-}$and half-life $388.2 \mathrm{~ms}$. The ordering in energy between the two states is given as unknown in NUBASE 2012 [59], although in Ref. [60] the ground state is assigned to $9 / 2^{-}$. The HFS of the ${ }^{197}$ At atomic transition at 216.225 $\mathrm{nm}$ used for the first excitation step of the RILIS ionization scheme [42] was measured by scanning the laser frequency while $\alpha$-particle counting using the "windmill"setup [61]. The details of the experiment and the resulting hyperfine structure analysis will be presented in a forthcoming publication. The positions of the HFS resonances of the two nuclear states are sufficiently separated to enable preferential ionization of the chosen isomer by an appropriate tuning of the laser frequency. An additional modification of the final ratio between the two states could be performed by exploiting their very different half-lives. Using a long trapping time in the ISOLTRAP cooler and buncher and a long excitation time in the precision trap, it was possible to favor the longer-lived, $1 / 2^{+}$state in the ToF-ICR measurement.

The measurements of the astatine ion beam were thus performed in two configurations. The final weights of the two states were determined for each configuration using the normalized hyperfine-structure measurements and taking into account the decay loss for each state. The first configuration used the first step laser of the RILIS tuned to the wave number of $15411.15 \mathrm{~cm}^{-1}$ (measured before the optical frequency tripling) and produced an initial weight of the $9 / 2^{-}$state of $93.3(6) \%$ (the rest being the $1 / 2^{+}$state). Using a total ISOLTRAP cycle time (translated to decay time) of 1082.8 $\mathrm{ms}$, the final weight of the $9 / 2^{-}$state in the ToF-ICR spectrum was $74.4(1.8) \%$ (the rest being the $1 / 2^{+}$state). The 


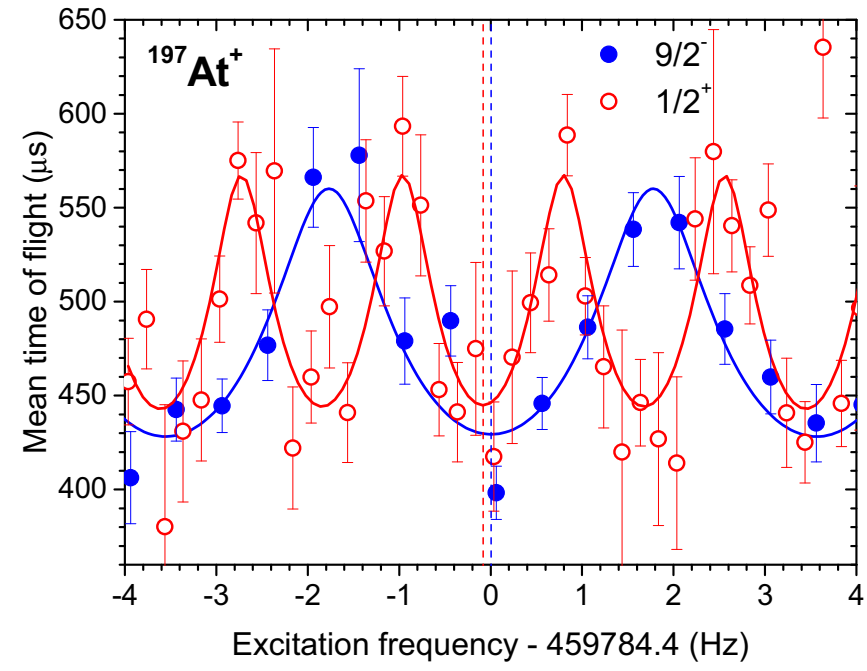

FIG. 3. Time-of-flight ion-cyclotron resonances of ${ }^{197} \mathrm{At}^{+}$, using a Ramsey-type excitation scheme [39,40]. The full blue symbols correspond to a weight of the $9 / 2^{-}$state in the measured beam of approximately $74.4 \%$, the rest being the $1 / 2^{+}$state. The open red symbols correspond to a weight of the $9 / 2^{-}$state of approximately $27.4 \%$. The fits by the theoretical line shapes are also shown. The dashed lines mark the centers of the two resonances.

cyclotron frequency ratio measured in this first configuration was $1.4822073444(930)$. The second configuration used a wave number of the first RILIS step of $15411.27 \mathrm{~cm}^{-1}$ and produced an initial weight of the $9 / 2^{-}$state of $84.8(7) \%$ (the rest being the $1 / 2^{+}$state). In this case, following a longer ISOLTRAP cycle time (translated to decay time) of 1872.8 $\mathrm{ms}$, the final weight of the $9 / 2^{-}$state in the ToF-ICR spectrum was reduced to $27.4(1.0) \%$ (the rest being the $1 / 2^{+}$state). The frequency ratio measured in this second configuration was 1.4822075175(608). An example of two Ramsey-type resonances measured in the two configurations is shown in Fig. 3.

A study of the variation of the measured frequencies with the number of ions simultaneously allowed in the precision trap detected no frequency shifts due to Coulomb interaction, within the statistical uncertainties. We consider that the measured resonance frequency in each spectrum is a weighted mean of the signals corresponding to the pure nuclear states, as suggested in Ref. [62] (see also Ref. [46]):

$$
v_{\text {res }}=w_{9 / 2^{-}} v_{c}^{9 / 2^{-}}+w_{1 / 2^{+}} v_{c}^{1 / 2^{+}}
$$

where $w$ denotes the weight of each nuclear state and $v_{c}$ is the corresponding cyclotron frequency. This assumption is correct in the limit of close, unresolved resonances, as is the case for ${ }^{197} \mathrm{At}^{+}$. By knowing the weights of the two states in the two configurations described above and the mixed frequency ratios, it is possible to perform a linear regression of the data set and extract the frequency ratios of the pure states. The final results obtained are given in Table II. The conclusion is that the $9 / 2^{-}$state is the nuclear ground state of ${ }^{197} \mathrm{At}$ and that the excitation energy of the $1 / 2^{+}$state is $45.6(22.8) \mathrm{keV}$. We note that the mass excess of the two states in ${ }^{197}$ At was previously determined based on $\alpha$-decay $Q$-value links $[60,63]$ to ${ }^{189} \mathrm{Tl}$
[48,51]. The determined excitation energy of the $1 / 2^{+}$state agrees with the value of 50(10) $\mathrm{keV}$ proposed in Ref. [60]. The result confirms that the $1 / 2^{+}$intruder state, interpreted as resulting from the promotion of a $s_{1 / 2}$ proton across the $Z=82$ gap to the $h_{9 / 2}$ single-particle level, is still higher in energy in ${ }^{197}$ At than the $9 / 2^{-}$state, which corresponds to three protons occupying $h_{9 / 2}$. The $1 / 2^{+}$intruder becomes the ground state in ${ }^{195} \mathrm{At}$, as determined in Ref. [65].

We note that in the determination of the initial weights of the two states we assumed equal $\alpha$ branching ratios. The $\alpha$ branching ratio of the $9 / 2^{-}$state is around $96 \%$, while that of the $1 / 2^{+}$is thought to be close to $100 \%$ [59]. A slightly different value of the two branching ratios would change the result of the linear regression of the two ${ }^{197} \mathrm{At}$ frequency ratios by a much smaller amount than their statistical uncertainties. Still, the discovery of a very different $\alpha$ branching ratio of the $1 / 2^{+}$state can be completely corrected for at a later time based on the information in the present work.

$$
\text { F. }{ }^{219} \mathrm{At}
$$

The beam of ${ }^{219} \mathrm{At}^{+}$was heavily contaminated by ${ }^{219} \mathrm{Fr}^{+}$. To purify the beam, the very short half-life of ${ }^{219} \mathrm{Fr}$ was exploited (20(2) ms [59]). The accumulation of ions in the ISOLTRAP cooler and buncher was synchronized to the impact of proton pulses on the ISOLDE target, with a delay of 400 ms. Additionally, a 400-ms buncher accumulation time was used for additional in-trap decay of the francium contamination. With these precautions, a pure ${ }^{219} \mathrm{At}^{+}$beam could be transported to the precision Penning trap. The determined mass agrees with the value from the AME 2012 [27], which is determined based on an $\alpha$-decay $Q$-value link [64].

\section{DISCUSSION}

The upper panel of Fig. 4 shows the experimental twoneutron separation energies of gold and mercury isotopes, compared to the mean-square charge radii of gold isotopes [66]. One notices the increase in charge radii at $N=107$, which marks the beginning of a region of deformation. The experimental $S_{2 N}$ values are represented for both the AME 2012 [27] data and for the masses measured in this work. One observes that with the ISOLTRAP masses of ${ }^{188,190} \mathrm{Au}$ the discontinuity in the $S_{2 N}$ trend around neutron number 110 is reduced, but not fully eliminated. This means that some nuclear structure effect might be at work. This could be connected to the onset of deformation at $N=107$; however, comparing to the $\delta\left\langle r^{2}\right\rangle$ of gold isotopes, we notice that the effect in $S_{2 N}$ takes place one neutron number higher.

To explain this difference, we represent in the lower panel of Fig. 4 the three-point estimator of the pairing gap in the gold and mercury isotopic chains, computed as

$$
\begin{aligned}
\Delta_{3 n}(Z, N)= & \frac{(-1)^{N}}{2}[E(Z, N-1) \\
& -2 E(Z, N)+E(Z, N+1)],
\end{aligned}
$$

where $E(Z, N)$ is the ground-state binding energy taken with negative sign [67]. 
It was already noted that the pairing gap decreases suddenly once the shape transition occurs in the gold chain at $N=107$ [68] and from Fig. 4 the correlation between the pairinggap and the charge-radii trends is exact. As discussed, for example, in Ref. [69], Eq. (1.16), the two-neutron separation energies cancel out partially the odd-even staggering of binding energies with neutron number, but they are sensitive to its first derivative. If one assumes the binding energy $E(Z, N)$ (negative sign) as composed of a smooth component corresponding to the trend of even-even isotopes $E_{0}(Z, N)$ and a term giving the odd-even staggering $\delta_{n}$, which is essentially a loss of binding due to the unpaired particle:

$$
E(Z, N)=E_{0}(Z, N)+\frac{(-1)^{N+1}+1}{2} \delta_{n}(Z, N),
$$

then the two-neutron separation energy will be given by

$$
\begin{aligned}
S_{2 N}(Z, N)= & E(Z, N-2)-E(Z, N) \\
= & E_{0}(Z, N-2)-E_{0}(Z, N) \\
& +\frac{(-1)^{N+1}+1}{2}\left[\delta_{n}(Z, N-2)-\delta_{n}(Z, N)\right] .
\end{aligned}
$$

This shows that on top of the trend given by the even-even isotopes, the $S_{2 N}$ values have an odd-even staggering of their own, given by the derivative of the odd-even staggering of the total binding energy. Going back to Fig. 4 (lower panel), one notices that between $N=109$ and $N=107$ there is a significant drop in $\Delta_{3 N}$, which according to Eq. (6) should lead to a reduction in $S_{2 N}$ at $N=109$ with respect to the trend of even-even isotopes. This agrees with what is observed in Fig. 4 (upper panel). The fact that no significant deviation is observed between $N=108$ and $N=106$ supports the idea that the phenomenon is connected to the effect of the odd neutron before and after the onset of deformation.

In order to discuss the evolution of two-neutron separation energies and charge radii with respect to the underlying picture of intrinsic nuclear shapes, including the effect of the unpaired nucleon, we will analyze the two observables in the framework of self-consistent mean-field theory. In the following, we will only summarize the main aspects of the calculations. More detailed information about the numerical approach are given in the Supplemental Material [70].

For this work, we performed HFB calculations [71,72] using the HFODD code $[73,74]$. The particle-hole part of the energy functional was computed using the SLy4 interaction [75], and the particle-particle part was computed using a $\delta$ pairing interaction of volume type [67], which is only characterized by the strength parameter $V_{0}$. The strength of the pairing interaction was adjusted to reproduce in the spherical approximation the odd-even staggering of binding energies in the lead isotopic chain, resulting in a value $V_{0}=-185 \mathrm{MeV}$ $\mathrm{fm}^{3}$ (see Ref. [70]).

For the calculations presented in this work, as for the ones performed for the region of neutron-rich nuclides of mass $A \approx 100$ in Ref. [79], we use a spherical basis, but we increase the maximum number of harmonic-oscillator shells to 500 , in order to improve the numerical description of the nuclear wave function. Calculations in optimized, axially deformed bases
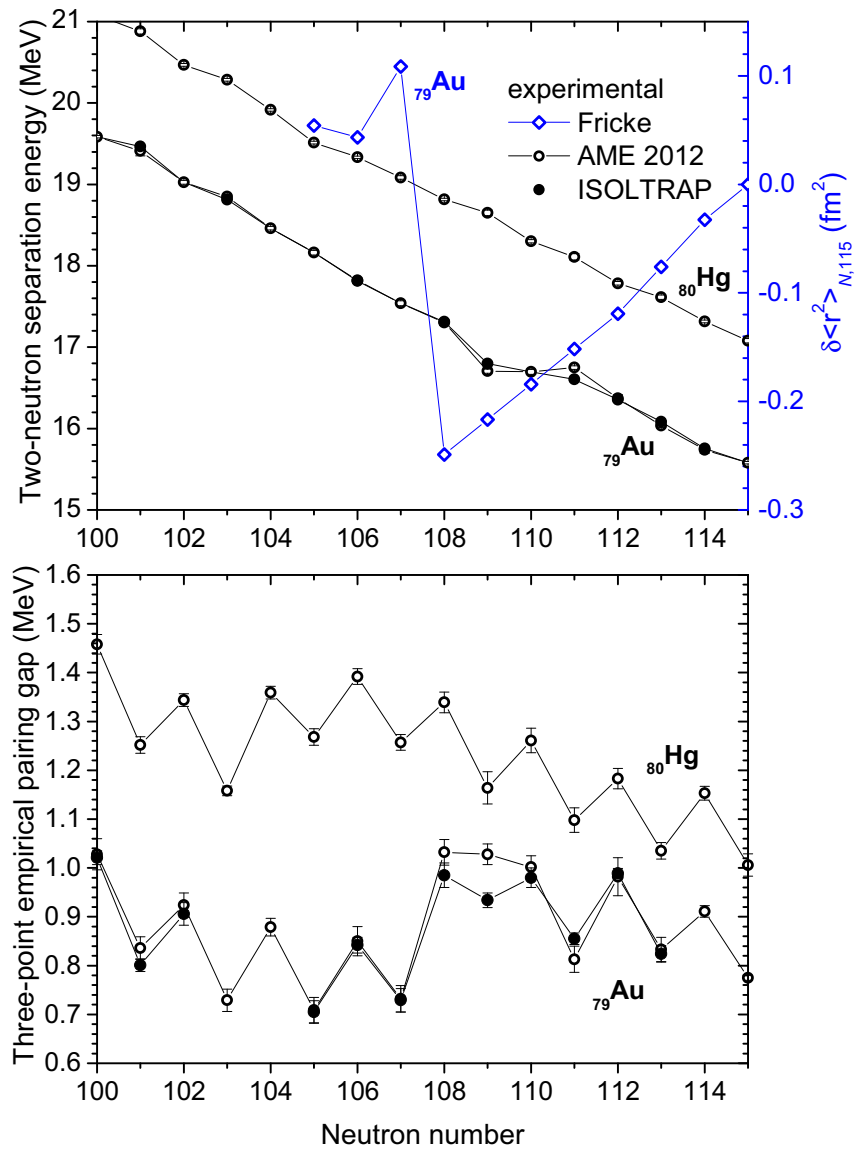

FIG. 4. Upper panel: experimental two-neutron separation energies for the gold and mercury isotopes compared to the mean-square charge radii of the gold isotopes. Lower panel: three-point empirical pairing gap for the mercury and gold isotopes in the region of interest. The experimental masses are from the atomic-mass evaluation [27] (empty symbols), this work (full symbols), and Ref. [29]. The mean-square charge radii are given as displacement to the $N=115$ value and taken from the Fricke evaluation [66].

were performed in Ref. [69], but suffer from an additional uncertainty related to the matching of the chosen basis to the deformation of each nucleus. We note, however, that the qualitative results are preserved in the present work.

In this work, the axial quadrupole-deformation degree of freedom was studied. The lowest stable configuration in energy is chosen as the ground state of the nucleus in the mean-field approximation. For discussing the evolution of nuclear structure along an isotopic chain, it is, however, also interesting to observe the properties of each equilibrium configuration found, even when it is not the lowest in energy. In the following, we will not only represent the trends of the ground-state observables, but also those corresponding to the lowest-energy prolate configuration and to the lowest-energy oblate configuration considered separately.

The results of a first such calculation are presented in Fig. 5, where the theoretical two-neutron separation energies and mean-square charge radii are compared for the gold and mercury isotopic chains to data from the atomic-mass evaluation [27] and this work (for the former) and the Fricke 

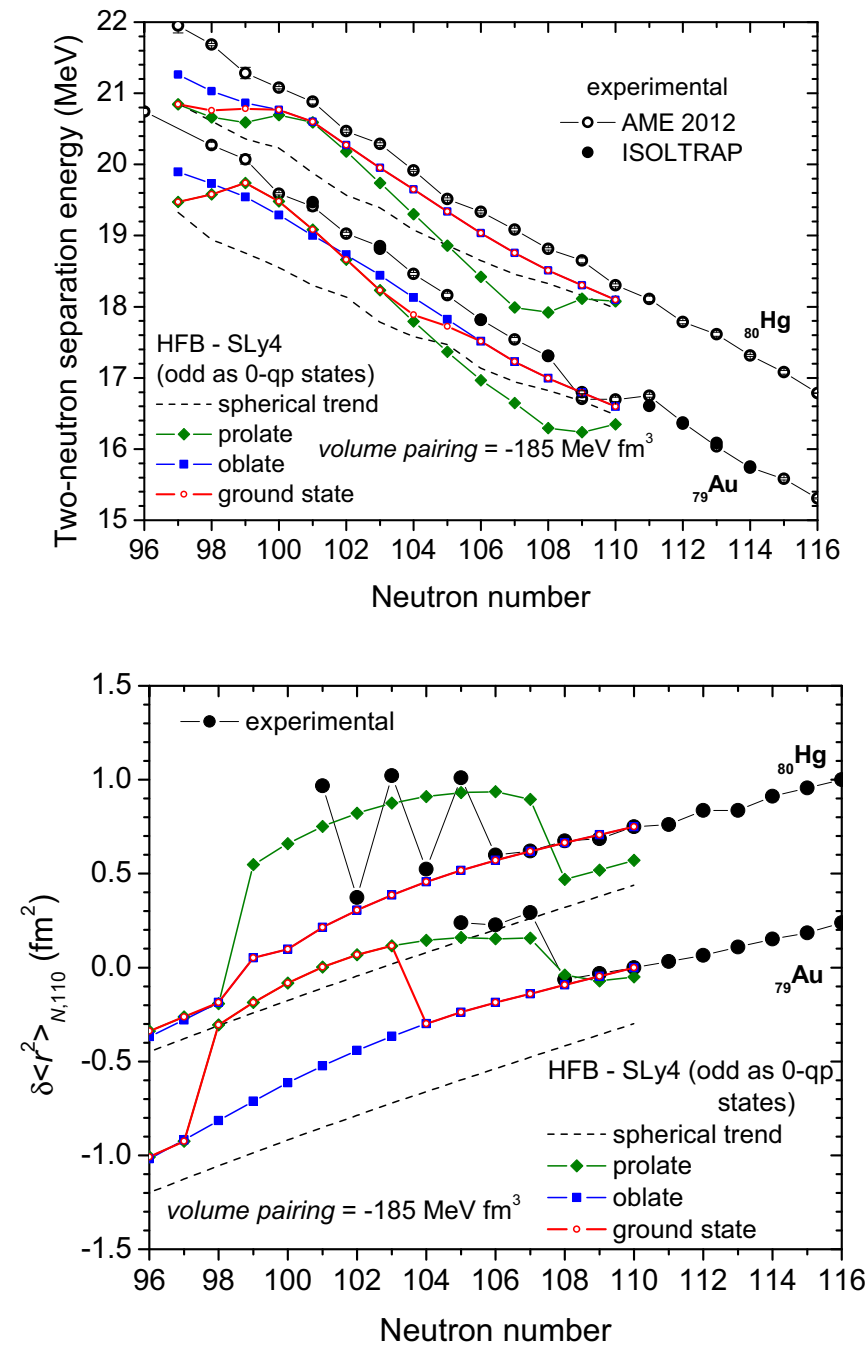

FIG. 5. Experimental two-neutron separation energies (upper panel) and mean-square charge radii (lower panel) for the gold and mercury isotopes, compared to the results of HFB calculations without quasiparticle blocking. The experimental masses are from the atomic-mass evaluation [27] (empty symbols), this work (full symbols), and Ref. [29]. The mean-square charge radii are given as displacement to the $N=110$ value and taken from the Fricke evaluation $[66,76]$. The calculations are presented separately for the lowest-energy oblate (blue squares) and prolate (green diamonds) solution, as well as for the ground state (empty red circles), assigned to the solution of lowest energy. The trends of the computed observables in a spherical HFB calculation are given with dashed lines. The two-neutron separation energies and charge radii of mercury isotopes are offset in order to better separate the two isotopic chains.

evaluation [66,76] (for the latter). In this case, odd and even nuclei were computed similarly as HFB zero-quasiparticle states (no blocking), meaning that in the odd and odd-odd systems all nucleons are paired, but form a wave function with odd number of constituents on average. This is possible in the HFB approach, because the Bogoliubov transformation does not conserve particle number. This calculation does not reproduce the odd-even staggering of binding energies, but gives a first-order trend of two-neutron separation energies and mean-square charge radii.

In Fig. 5, one notices that the experimental mean-square charge radii of mercury isotopes also exhibit a significant change of trend below neutron number 107, entering a region of strong odd-even staggering. On the other hand, no significant change of trend in the two-neutron separation energies is observed around the transition in the mercury chain. The calculations in Fig. 5 show that the mean field of the gold and mercury isotopes has two important coexisting nuclear configurations, of which one has oblate and the other has prolate quadrupole deformation. The evolution of the two configurations along the isotopic chains is readily observed in the mean-square charge radii. For both chains, the prolate configuration undergoes a jump at $N=107$ from low to high deformation, while the oblate configuration has a smooth evolution with neutron number. A similar picture of axial intrinsic oblate and prolate shapes was presented recently in the study of $\beta$-decay properties of mercury and platinum isotopes by QRPA calculations built on a HF+BCS basis [77] and the impact on the charge radii was discussed.

In the gold isotopic chain, the oblate configuration is more bound for $N>103$ than the prolate one. At $N=103$, however, the prolate configuration becomes the lowest in energy. This produces a clear jump in the ground-state charge radii. Still, due to the fact that the oblate configuration has a smooth trend before the transition point and the transition is not accompanied by a sudden change in energy, there is only a weak change in the trend of two-neutron separation energies around the transition point. In the mercury isotopic chain, the calculations without blocking predict that the oblate configuration should remain the ground state all along the isotopic chain and thus no jump in charge radii should occur, at odds with experimental evidence.

The HFB wave function of odd nuclei must, however, be described by taking into account that the unpaired nucleon does not contribute to the pairing field. This is achieved in the blocking approximation, which means more specifically that the ansatz for the odd nuclei is not taken as the HFB zero-quasiparticle state but a one-quasiparticle excitation on the HFB vacuum, as described in Refs. [73,74].

By the blocking mechanism, the odd nucleus loses binding with respect to the fully paired solution (the latter is also called false vacuum [78]). The amount of energy lost for each isotope is also called "blocking gap" [67] and will affect the energy balance between the prolate and oblate solutions. Interestingly, we find that in each of the two isotopic chains the two configurations lose systematically different amounts of energy in the region of interest $(N<110)$. More specifically, the prolate configuration loses less energy than the oblate one by the blocking of the odd nucleon. The impact on the energy balance is illustrated in Fig. 6 for the mercury chain, where the difference between the binding energy of the prolate and oblate solutions is shown for each isotope. Without blocking (black squares), the difference is positive for almost all isotopes, which means that the prolate solution is always less bound and the ground state is oblate. The interchange between the two only takes place at $N=97$, when the prolate configuration has already lost its large deformation, so no major change in 


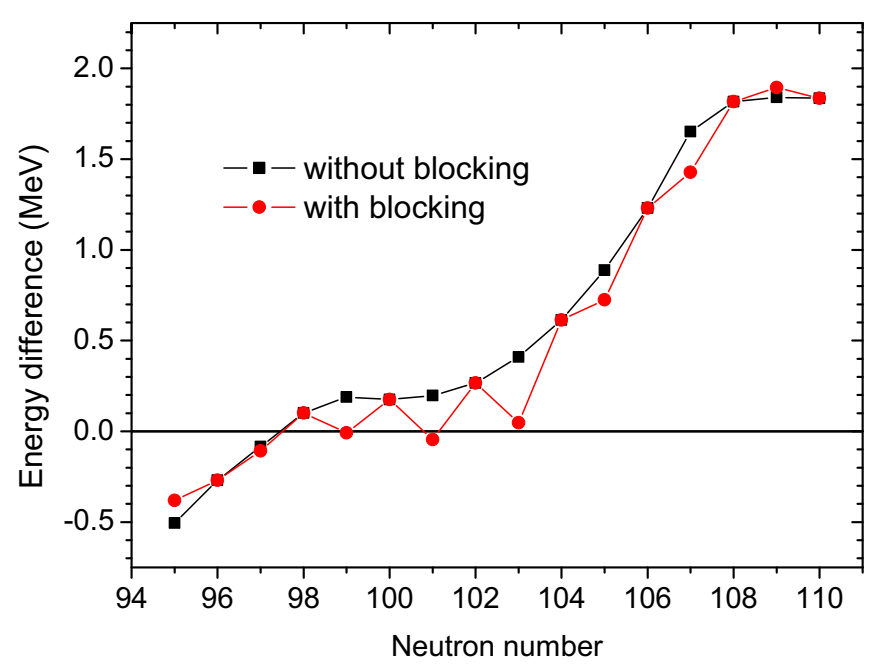

FIG. 6. Difference in binding energy between the prolate and the oblate mean-field configuration for a calculation without quasiparticle blocking (black squares) and with quasiparticle blocking (red circles) for mercury isotopes. Negative values correspond to nuclei for which the prolate configuration is more bound.

the trend of the charge radii is predicted. This corresponds to the situation represented in Fig. 5. With blocking, however, the binding-energy differences of the odd mercury isotopes are shifted on a different trend, approaching 0 , or even crossing it, in the region of large prolate deformation. For the odd isotopes for which the crossing occurs, an oblate to prolate transition takes place in the ground state. For these isotopes the charge radius should oscillate as a function of neutron number between the oblate value (in the even systems) and prolate value (in the odd systems).

The consequence of blocking at the level of observables is illustrated in Fig. 7, where the mean-square charge radii and two-neutron separation energies are again represented. For the gold isotopic chain, proton blocking was implemented for the even- $N$ isotopes, but the odd-odd isotopes are not calculated. For the odd- $N$ mercury isotopes, neutron blocking was implemented. One notices for the mercury chain that the trend of mean-square charge radii now shows the strong odd-even staggering observed in experiment (although displaced to more neutron-deficient isotopes). The trend of twoneutron separation energies remains relatively smooth after quasiparticle blocking is implemented, although in the gold isotopic chain the change of trend around the oblate-prolate transition point is slightly more pronounced than in Fig. 5. We note that the difference between the two configurations in the region of large prolate deformation is below the typical precision of mean-field calculations [6,7], so although the pairing interaction was not tuned specifically to reproduce the odd-even staggering of mercury charge radii, a slightly different value would have shifted the differences enough to maintain the oblate configuration as the ground state, or make the crossing even more pronounced (for example, already taking place at $N=103$, as is almost the case in Fig. 6). Still, the qualitative conclusion of Fig. 6, specifically the fact that the prolate configuration loses less energy by blocking than the
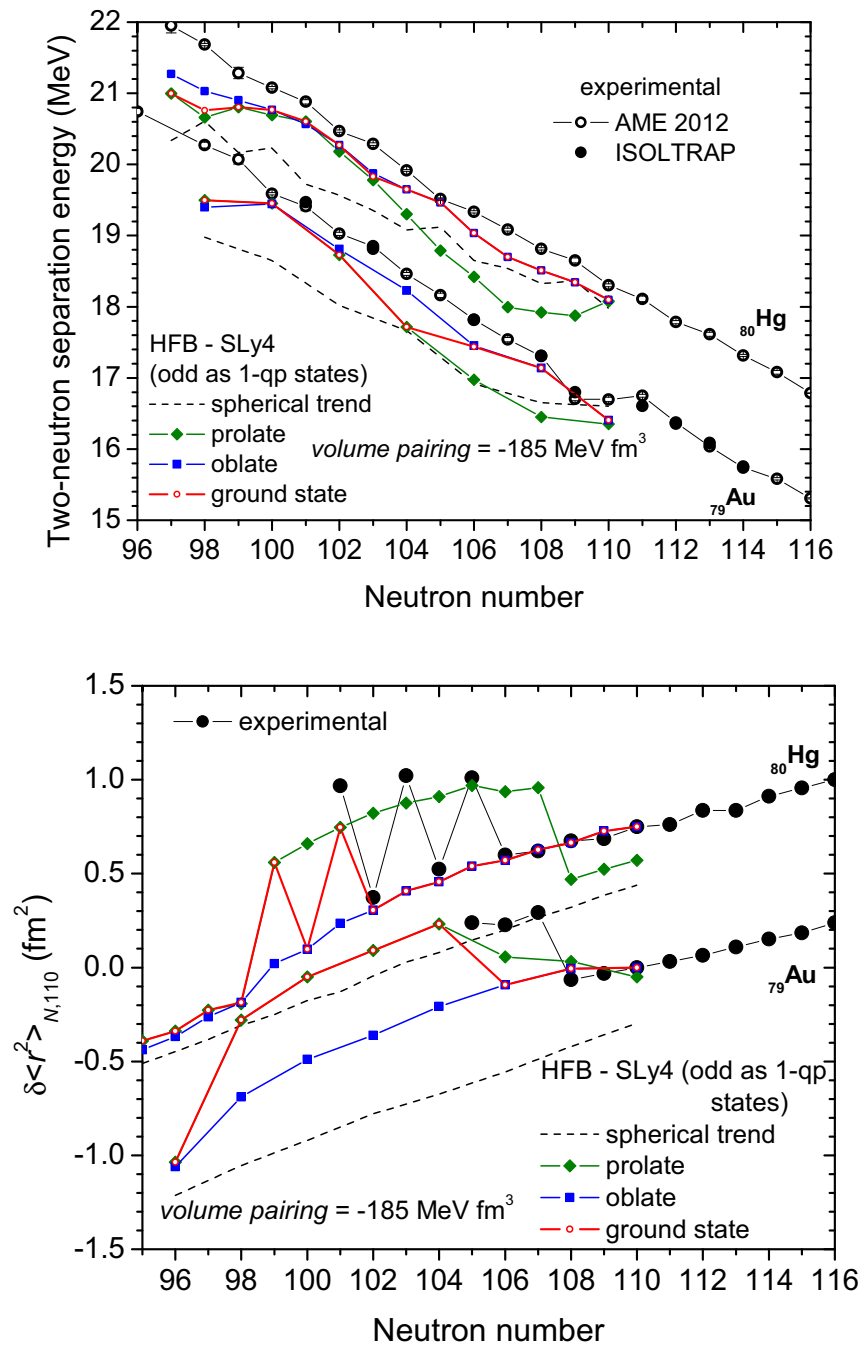

FIG. 7. Same as Fig. 5, but the calculations include proton quasiparticle blocking for the gold isotopes of even neutron number and neutron quasiparticle blocking for the odd mercury isotopes.

oblate one in the region of large deformation, is robust against a readjustment of the pairing interaction.

\section{CONCLUSIONS AND OUTLOOK}

In this work, we reported on ISOLTRAP mass measurements of neutron-deficient gold isotopes, as well as the masses of two astatine isotopes and one isomer. The isotopes were produced by the ISOLDE facility at CERN. Significant deviations from the atomic-mass evaluation [27] were found for ${ }^{188} \mathrm{Au}$ and ${ }^{190} \mathrm{Au}$, which partially smooths out the experimental trend of two-neutron separation energies, although not completely, highlighting the important role of subtle changes in binding energies and hence illustrating the interest of high-precision measurements. The mass excess and ordering of the two long-lived states in ${ }^{197}$ At were determined directly by a combination of selective laser ionization and mass spectrometry.

The measured neutron-deficient isotopes are situated in a well-known region of shape coexistence [14], in which nuclear 
charge radii exhibit spectacular jumps (in the gold isotopic chain) and staggering (in the mercury isotopic chain). The new measured masses suggest a weaker sensitivity of the mass surface to these phenomena than initially thought; however, through the reduction of the odd-even staggering in the region of deformation some effect is preserved in both the gold and the mercury isotopic chains, as also discussed in Ref. [68]. In the gold isotopic chain, this effect is strong enough to leave some staggering also in the two-neutron separation energy; however, in the mercury isotopic chain it is reduced.

To explain the difference, we performed HFB calculations with the HFODD code [73,74], the SLy4 interaction [75], and a $\delta$ volume pairing interaction. Using a pairing interaction adjusted to describe the odd-even staggering of masses in the lead isotopic chain (computed in the spherical approximation), it was possible to describe qualitatively the changes in charge radii, as well as the lower sensitivity of the mass surface, although the two-neutron separation energies are systematically lower and the jumps in charge radii are predicted to take place for lower neutron number than observed experimentally. It was shown that the phenomena arise from the balance in energy between oblate and prolate configurations along the isotopic chains. It was also illustrated that in the quasiparticle blocking approximation, the prolate configurations lose less energy (have lower blocking gap) than the oblate ones. This leads to a slightly different picture of the nuclear configurations once blocking is implemented, manifested in the mercury chain by a successive shift between the oblate and the prolate configurations of the nuclear mean field. In turn, this produces an effect which qualitatively resembles what is observed experimentally.

As discussed in Ref. [79] for the neutron-rich nuclides of mass $A \approx 100$, the neutron-deficient lead region offers another example in which properties of midshell nuclei can be used to constrain mean-field or density-functional-type approaches. Experimental signatures such as the increase in two-neutron separation energies and charge radii at $N=60$ in the isotopic chains between rubidium and zirconium and the strong oddeven staggering of charge radii of neutron-deficient mercury isotopes are predicted to occur in the mean-field theory. However, their position and strength require a specific energy balance between the competing mean-field configurations, which narrows down the range of values that the parameters of the model can take (one example being the strength of the pairing interaction). Adding these specific regions and properties to the input used to adjust phenomenological models can improve their overall description and predictive power of nuclear properties.

A treatment of nuclear pairing and quadrupole correlations beyond the mean field is necessary to check which other contributions to the ground-state binding energy can change the picture drawn so far. The conclusions concerning the return to less deformed states on the very neutron-deficient side of the gold and mercury isotopic chains agree with results of other global mean-field calculations, which include beyond meanfield correlations $[80,81]$. It is thus of particular interest to determine whether the mass surface will remain smooth further than the region so far accessible by direct measurements, or whether the end of the region of nuclear collectivity will produce more visible signatures. Beyond-mean-field calculations of odd nuclei are also a point of great general interest, as currently they are only applied to selected cases [82].

\section{ACKNOWLEDGMENTS}

This project has received funding through the European Union's Seventh Framework Programme for Research and Technological Development under Grant Agreements No. 262010 (ENSAR), No. 267194 (COFUND), and No. 289191 (LA ${ }^{3} \mathrm{NET}$ ); from the Bundesministerium für Bildung und Forschung under Contracts No. 05P15HGCIA, No. 05P12HGCI1, No. 05P12HGFNE, and No. 05P09ODCIA; the French IN2P3; the ISOLDE Collaboration; and the Max-Planck Society. S.K. acknowledges support from the Robert-Bosch Foundation. R.B.C. acknowledges support from the Partner Group with the Max-Planck Society. We would like to thank the ISOLDE target and operations teams for their support, as well as the Windmill team for identifying the isomeric beam of ${ }^{197} \mathrm{At}^{+}$. V.M. would like to thank J. Dobaczewski and A. N. Andreyev for discussions and suggestions to the manuscript.
[1] R. Roth, S. Binder, K. Vobig, A. Calci, J. Langhammer, and P. Navrátil, Phys. Rev. Lett. 109, 052501 (2012).

[2] G. Hagen, T. Papenbrock, M. Hjorth-Jensen, and D. J. Dean, Rep. Prog. Phys. 77, 096302 (2014).

[3] V. Somà, A. Cipollone, C. Barbieri, P. Navrátil, and T. Duguet, Phys. Rev. C 89, 061301 (2014).

[4] H. Hergert, S. Bogner, T. Morris, A. Schwenk, and K. Tsukiyama, Phys. Rep. 621, 165 (2016).

[5] E. Caurier, G. Martínez-Pinedo, F. Nowacki, A. Poves, and A. P. Zuker, Rev. Mod. Phys. 77, 427 (2005).

[6] Y. Gao, J. Dobaczewski, M. Kortelainen, J. Toivanen, and D. Tarpanov, Phys. Rev. C 87, 034324 (2013).

[7] J. D. McDonnell, N. Schunck, D. Higdon, J. Sarich, S. M. Wild, and W. Nazarewicz, Phys. Rev. Lett. 114, 122501 (2015).
[8] M. Mumpower, R. Surman, G. McLaughlin, and A. Aprahamian, Progr. Part. Nucl. Phys. 86, 86 (2016).

[9] T. Duguet, H. Hergert, J. D. Holt, and V. Somà, Phys. Rev. C 92, 034313 (2015).

[10] S. Bogner, R. Furnstahl, and A. Schwenk, Progr. Part. Nucl. Phys. 65, 94 (2010).

[11] S. Bogner, A. Bulgac, J. Carlson, J. Engel, G. Fann, R. Furnstahl, S. Gandolfi, G. Hagen, M. Horoi, C. Johnson, M. Kortelainen, E. Lusk, P. Maris, H. Nam, P. Navratil, W. Nazarewicz, E. Ng, G. Nobre, E. Ormand, T. Papenbrock, J. Pei, S. Pieper, S. Quaglioni, K. Roche, J. Sarich, N. Schunck, M. Sosonkina, J. Terasaki, I. Thompson, J. Vary, and S. Wild, Comput. Phys. Commun. 184, 2235 (2013). 
[12] R. B. Cakirli, R. F. Casten, and K. Blaum, Phys. Rev. C 82, 061306 (2010).

[13] A. Andreyev, M. Huyse, P. Van Duppen, L. Weissman, D. Ackermann, J. Gerl, F. P. Hessberger, S. Hofmann, A. Kleinböhl, G. Münzenberg, S. Reshitko, C. Schlegel, H. Schaffner, P. Cagarda, M. Matos, S. Saro, A. Keenan, C. Moore, C. D. O'Leary, R. D. Page, M. Taylor, H. Kettunen, M. Leino, A. Lavrentiev, R. Wyss, and K. Heyde, Nature (London) 405, 430 (2000).

[14] K. Heyde and J. L. Wood, Rev. Mod. Phys. 83, 1467 (2011).

[15] H. De Witte, A. N. Andreyev, N. Barré, M. Bender, T. E. Cocolios, S. Dean, D. Fedorov, V. N. Fedoseyev, L. M. Fraile, S. Franchoo, V. Hellemans, P. H. Heenen, K. Heyde, G. Huber, M. Huyse, H. Jeppessen, U. Köster, P. Kunz, S. R. Lesher, B. A. Marsh, I. Mukha, B. Roussière, J. Sauvage, M. Seliverstov, I. Stefanescu, E. Tengborn, K. Van de Vel, J. Van de Walle, P. Van Duppen, and Y. Volkov, Phys. Rev. Lett. 98, 112502 (2007).

[16] K. Wallmeroth, G. Bollen, A. Dohn, P. Egelhof, J. Grüner, F. Lindenlauf, U. Krönert, J. Campos, A. Rodriguez Yunta, M. J. G. Borge, A. Venugopalan, J. L. Wood, R. B. Moore, and H. J. Kluge, Phys. Rev. Lett. 58, 1516 (1987).

[17] K. Wallmeroth, G. Bollen, A. Dohn, P. Egelhof, U. Krönert, M. Borge, J. Campos, A. Yunta, K. Heyde, C. De Coster, J. Wood, and H.-J. Kluge, Nucl. Phys. A 493, 224 (1989).

[18] G. Savard, J. Crawford, J. Lee, G. Thekkadath, H. Duong, J. Pinard, F. Le Blanc, P. Kilcher, J. Obert, J. Oms, J. Putaux, B. Roussiere, and J. Sauvage, Nucl. Phys. A 512, 241 (1990).

[19] F. Le Blanc, J. Obert, J. Oms, J. C. Putaux, B. Roussière, J. Sauvage, J. Pinard, L. Cabaret, H. T. Duong, G. Huber, M. Krieg, V. Sebastian, J. Crawford, J. K. P. Lee, J. Genevey, and F. Ibrahim, Phys. Rev. Lett. 79, 2213 (1997).

[20] C. Böhm, C. Borgmann, G. Audi, D. Beck, K. Blaum, M. Breitenfeldt, R. B. Cakirli, T. E. Cocolios, S. Eliseev, S. George, F. Herfurth, A. Herlert, M. Kowalska, S. Kreim, D. Lunney, V. Manea, E. Minaya Ramirez, S. Naimi, D. Neidherr, M. Rosenbusch, L. Schweikhard, J. Stanja, M. Wang, R. N. Wolf, and K. Zuber, Phys. Rev. C 90, 044307 (2014).

[21] A. E. Barzakh, L. K. Batist, D. V. Fedorov, V. S. Ivanov, K. A. Mezilev, P. L. Molkanov, F. V. Moroz, S. Y. Orlov, V. N. Panteleev, and Y. M. Volkov, Phys. Rev. C 88, 024315 (2013).

[22] J. Bonn, G. Huber, H.-J. Kluge, L. Kugler, and E. Otten, Phys. Lett. B 38, 308 (1972).

[23] J. Bonn, G. Huber, H.-J. Kluge, and E. Otten, Z. Phys. A 276, 203 (1976).

[24] T. Kühl, P. Dabkiewicz, C. Duke, H. Fischer, H. J. Kluge, H. Kremmling, and E. W. Otten, Phys. Rev. Lett. 39, 180 (1977).

[25] G. Ulm, S. Bhattacherjee, P. Dabkiewicz, G. Huber, H.-J. Kluge, T. Kühl, H. Lochmann, E.-W. Otten, K. Wendt, S. Ahmad, W. Klempt, R. Neugart, and ISOLDE Collaboration, Z. Phys. A 325, 247 (1986)

[26] N. Bree, K. Wrzosek-Lipska, A. Petts, A. Andreyev, B. Bastin, M. Bender, A. Blazhev, B. Bruyneel, P. A. Butler, J. Butterworth, M. P. Carpenter, J. Cederkäll, E. Clément, T. E. Cocolios, A. Deacon, J. Diriken, A. Ekström, C. Fitzpatrick, L. M. Fraile, C. Fransen, S. J. Freeman, L. P. Gaffney, J. E. García-Ramos, K. Geibel, R. Gernhäuser, T. Grahn, M. Guttormsen, B. Hadinia, K. K. Hadyńska-Kle, M. Hass, P.-H. Heenen, R.-D. Herzberg, H. Hess, K. Heyde, M. Huyse, O. Ivanov, D. G. Jenkins, R. Julin, N. Kesteloot, T. Kröll, R. Krücken, A. C. Larsen, R. Lutter, P. Marley, P. J. Napiorkowski, R. Orlandi, R. D. Page, J. Pakarinen, N. Patronis, P. J. Peura, E. Piselli, P. Rahkila, E. Rapisarda, P.
Reiter, A. P. Robinson, M. Scheck, S. Siem, K. Singh Chakkal, J. F. Smith, J. Srebrny, I. Stefanescu, G. M. Tveten, P. Van Duppen, J. Van de Walle, D. Voulot, N. Warr, F. Wenander, A. Wiens, J. L. Wood, and M. Zielinska, Phys. Rev. Lett. 112, 162701 (2014).

[27] M. Wang, G. Audi, A. H. Wapstra, F. G. Kondev, M. MacCormick, X. Xu, and B. Pfeiffer, Chin. Phys. C 36, 1603 (2012).

[28] M. Mukherjee, D. Beck, K. Blaum, G. Bollen, J. Dilling, S. George, F. Herfurth, A. Herlert, A. Kellerbauer, H.-J. Kluge, S. Schwarz, L. Schweikhard, and C. Yazidjian, Eur. Phys. J. A 35, 1 (2008).

[29] S. Kreim, D. Atanasov, D. Beck, K. Blaum, C. Böhm, C. Borgmann, M. Breitenfeldt, T. Cocolios, D. Fink, S. George, A. Herlert, A. Kellerbauer, U. Köster, M. Kowalska, D. Lunney, V. Manea, E. Minaya Ramirez, S. Naimi, D. Neidherr, T. Nicol, R. Rossel, M. Rosenbusch, L. Schweikhard, J. Stanja, F. Wienholtz, R. Wolf, and K. Zuber, Nucl. Instr. Meth. B 317, 492 (2013).

[30] E. Kugler, Hyperfine Interact. 129, 23 (2000).

[31] V. N. Fedosseev, L.-E. Berg, D. V. Fedorov, D. Fink, O. J. Launila, R. Losito, B. A. Marsh, R. E. Rossel, S. Rothe, M. D. Seliverstov, A. M. Sjödin, and K. D. A. Wendt, Rev. Sci. Instrum. 83, 02A903 (2012).

[32] R. N. Wolf, F. Wienholtz, D. Atanasov, D. Beck, K. Blaum, C. Borgmann, F. Herfurth, M. Kowalska, S. Kreim, Y. A. Litvinov, D. Lunney, V. Manea, D. Neidherr, M. Rosenbusch, L. Schweikhard, J. Stanja, and K. Zuber, Int. J. Mass Spectrom. 349-350, 123 (2013).

[33] R. N. Wolf, D. Beck, K. Blaum, C. Böhm, C. Borgmann, M. Breitenfeldt, F. Herfurth, A. Herlert, M. Kowalska, S. Kreim, D. Lunney, S. Naimi, D. Neidherr, M. Rosenbusch, L. Schweikhard, J. Stanja, F. Wienholtz, and K. Zuber, Nucl. Instr. Meth. A 686, 82 (2012).

[34] N. E. Bradbury and R. A. Nielsen, Phys. Rev. 49, 388 (1936).

[35] F. Wienholtz, D. Atanasov, S. Kreim, V. Manea, M. Rosenbusch, L. Schweikhard, A. Welker, and R. N. Wolf, Phys. Scr. T166, 014068 (2015).

[36] H. Raimbault-Hartmann, D. Beck, G. Bollen, M. König, H.-J. Kluge, E. Schark, J. Stein, S. Schwarz, and J. Szerypo, Nucl. Instr. Meth. B 126, 378 (1997).

[37] G. Savard, S. Becker, G. Bollen, H.-J. Kluge, R. Moore, T. Otto, L. Schweikhard, H. Stolzenberg, and U. Wiess, Phys. Lett. A 158, 247 (1991).

[38] M. König, G. Bollen, H.-J. Kluge, T. Otto, and J. Szerypo, Int. J. Mass Spectrom. 142, 95 (1995).

[39] S. George, S. Baruah, B. Blank, K. Blaum, M. Breitenfeldt, U. Hager, F. Herfurth, A. Herlert, A. Kellerbauer, H.-J. Kluge, M. Kretzschmar, D. Lunney, R. Savreux, S. Schwarz, L. Schweikhard, and C. Yazidjian, Phys. Rev. Lett. 98, 162501 (2007).

[40] S. George, K. Blaum, F. Herfurth, A. Herlert, M. Kretzschmar, S. Nagy, S. Schwarz, L. Schweikhard, and C. Yazidjian, Int. J. Mass Spectrom. 264, 110 (2007).

[41] B. A. Marsh, V. N. Fedosseev, and P. Kosuri, Hyperfine Interact. 171, 109 (2006).

[42] S. Rothe, A. N. Andreyev, S. Antalic, A. Borschevsky, L. Capponi, T. E. Cocolios, H. De Witte, E. Eliav, D. V. Fedorov, V. N. Fedosseev, D. A. Fink, S. Fritzsche, L. Ghys, M. Huyse, N. Imai, U. Kaldor, Y. Kudryavtsev, U. Köster, J. F. W. Lane, J. Lassen, V. Liberati, K. M. Lynch, B. A. Marsh, K. Nishio, D. Pauwels, V. Pershina, L. Popescu, T. J. Procter, D. Radulov, S. Raeder, M. M. Rajabali, E. Rapisarda, R. E. Rossel, K. Sandhu, 
M. D. Seliverstov, A. M. Sjödin, P. Van den Bergh, P. Van Duppen, M. Venhart, Y. Wakabayashi, and K. D. A. Wendt, Nat. Commun. 4, 1835 (2013).

[43] S. Rothe, V. N. Fedosseev, T. Kron, B. A. Marsh, R. E. Rossel, and K. D. A. Wendt, Nucl. Instrum. Methods Phys. Res., Sect. B 317, 561 (2013).

[44] V. N. Fedosseev, D. V. Fedorov, R. Horn, G. Huber, U. Köster, J. Lassen, V. I. Mishin, M. D. Seliverstov, L. Weissman, and K. Wendt, Nucl. Instr. Methods Phys. Res., Sec.. B 204, 353 (2003).

[45] B. A. Marsh, B. Andel, A. N. Andreyev, S. Antalic, D. Atanasov, A. E. Barzakh, B. Bastin, Ch. Borgmann, L. Capponi, T. E. Cocolios, T. Day Goodacre, M. Dehairs, X. Derkx, H. De Witte, D. V. Fedorov, V. N. Fedosseev, G. J. Focker, D. A. Fink, K. T. Flanagan, S. Franchoo, L. Ghys, M. Huyse, N. Imai, Z. Kalaninova, U. Köster, S. Kreim, N. Kesteloot, Yu. Kudryavtsev, J. Lane, N. Lecesne, V. Liberati, D. Lunney, K. M. Lynch, V. Manea, P. L. Molkanov, T. Nicol, D. Pauwels, L. Popescu, D. Radulov, E. Rapisarda, M. Rosenbusch, R. E. Rossel, S. Rothe, L. Schweikhard, M. D. Seliverstov, S. Sels, A. M. Sjödin, V. Truesdale, C. Van Beveren, P. Van Duppen, K. Wendt, F. Wienholtz, R. N. Wolf, and S. G. Zemlyanoy, Nucl. Instrum. Methods Phys. Res., Sect. B 317, 550 (2013).

[46] A. Kellerbauer, K. Blaum, G. Bollen, F. Herfurth, H.-J. Kluge, M. Kuckein, E. Sauvan, C. Scheidenberger, and L. Schweikhard, Eur. Phys. J. D 22, 53 (2003).

[47] A. Neskakis, R. Lieder, H. Beuscher, Y. Gono, D. Haenni, and M. Müller-Veggian, Nucl. Phys. A 390, 53 (1982).

[48] Y. Litvinov, H. Geissel, T. Radon, F. Attallah, G. Audi, K. Beckert, F. Bosch, M. Falch, B. Franzke, M. Hausmann, M. Hellström, T. Kerscher, O. Klepper, H.-J. Kluge, C. Kozhuharov, K. Löbner, G. Münzenberg, F. Nolden, Y. Novikov, W. Quint, Z. Patyk, H. Reich, C. Scheidenberger, B. Schlitt, M. Steck, K. Sümmerer, L. Vermeeren, M. Winkler, T. Winkler, and H. Wollnik, Nuc. Phys. A 756, 3 (2005).

[49] A. Johansson and B. Nyman, Phys. Scr. 8, 99 (1973).

[50] J. S. Dionisio and C. Vieu, in International Conference on Nuclear Structure and Spectroscopy, Amsterdam, 1974 (Grüner, Amsterdam, 1974).

[51] T. Radon, H. Geissel, G. Münzenberg, B. Franzke, T. Kerscher, F. Nolden, Y. Novikov, Z. Patyk, C. Scheidenberger, F. Attallah, K. Beckert, T. Beha, F. Bosch, H. Eickhoff, M. Falch, Y. Fujita, M. Hausmann, F. Herfurth, H. Irnich, H. Jung, O. Klepper, C. Kozhuharov, Y. Litvinov, K. Löbner, F. Nickel, H. Reich, W. Schwab, B. Schlitt, M. Steck, K. Sümmerer, T. Winkler, and H. Wollnik, Nucl. Phys. A 677, 75 (2000).

[52] H. Dautet, N. Campeau, J. K. P. Lee, C. Bourgeois, B. Roussiere, and A. Houdayer, in International Conference on Atomic Masses and Fundamental Constants, Seeheim-Jugenheim, Germany (AMCO-7), 1984 (Technische Hochschule, Darmstadt, 1984).

[53] G. Albouy, M. M. Gusakow, and N. Poffe, J. Phys. Radium 21, 751 (1960).

[54] M. Finger, R. Foucher, J. P. Husson, J. Jastrzebski, A. Johnson, C. Sebille, R. Henck, J. M. Kuchly, R. Regal, P. Siffert, G. Astner, B. R. Erdal, E. Hagebo, A. Kjelberg, F. Munnich, P. Patzelt, E. Beck, and H. Kugler, Main Decay Characteristics of Mercury Isotopes and Their Daughter Products $(182<\mathrm{A}<$ 192), Tech. Rep. CERN 70-29, 1970 (unpublished).

[55] H. Dautet, R. Turcotte, and S. K. Mark, Annual Report of McGill University, Canada, 1986 (unpublished).

[56] A. Siivola, Nucl. Phys. A 109, 231 (1968).
[57] J. Keller, K.-H. Schmidt, F. Hessberger, G. Münzenberg, W. Reisdorf, H.-G. Clerc, and C.-C. Sahm, Nucl. Phys. A 452, 173 (1986).

[58] K. Toth, M. Ijaz, J. Lin, E. Robinson, B. Hannah, E. Spejewski, J. Cole, J. Hamilton, and A. Ramayya, Phys. Lett. B 63, 150 (1976)

[59] G. Audi, F. Kondev, M. Wang, B. Pfeiffer, X. Sun, J. Blachot, and M. MacCormick, Chin. Phys. C 36, 1157 (2012).

[60] E. Coenen, K. Deneffe, M. Huyse, P. Van Duppen, and J. L. Wood, Z. Phys. A 324, 485 (1986).

[61] M. D. Seliverstov, T. E. Cocolios, W. Dexters, A. N. Andreyev, S. Antalic, A. E. Barzakh, B. Bastin, J. Büscher, I. G. Darby, D. V. Fedorov, V. N. Fedosseev, K. T. Flanagan, S. Franchoo, G. Huber, M. Huyse, M. Keupers, U. Köster, Y. Kudryavtsev, B. A. Marsh, P. L. Molkanov, R. D. Page, A. M. Sjödin, I. Stefan, P. Van Duppen, M. Venhart, and S. G. Zemlyanoy, Phys. Rev. C 89, 034323 (2014).

[62] G. Audi, M. Wang, A. H. Wapstra, F. G. Kondev, M. MacCormick, X. Xu, and B. Pfeiffer, Chin. Phys. C 36, 1287 (2012).

[63] W. Treytl and K. Valli, Nucl. Phys. A 97, 405 (1967).

[64] C. F. Liang, P. Paris, and R. K. Sheline, Phys. Rev. C 64, 034310 (2001)

[65] H. Kettunen, T. Enqvist, M. Leino, K. Eskola, P. Greenlees, K. Helariutta, P. Jones, R. Julin, S. Juutinen, H. Kankaanpää, H. Koivisto, P. Kuusiniemi, M. Muikku, P. Nieminen, P. Rahkila, and J. Uusitalo, Eur. Phys. J. A 16, 457 (2003).

[66] G. Fricke and K. Heilig, in Nuclear Charge Radii, LandoltBrnstein Group I Elementary Particles, Nuclei, and Atoms, Vol. 20, edited by H. Schopper (Springer, Berlin, 2004), Chap. 79, pp. 1-4.

[67] M. Bender, K. Rutz, P.-G. Reinhard, and J. Maruhn, Eur. Phys. J. A 8, 59 (2000).

[68] C. Weber, G. Audi, D. Beck, K. Blaum, G. Bollen, F. Herfurth, A. Kellerbauer, H.-J. Kluge, D. Lunney, and L. Schweikhard, Nucl. Phys. A 803, 1 (2008).

[69] V. Manea, Penning-trap mass measurements of exotic rubidium and gold isotopes for a mean-field study of pairing and quadrupole correlations, Ph.D. thesis, Université Paris-Sud, Orsay, France, 2014, https://tel.archives-ouvertes.fr/tel-01093204/.

[70] See Supplemental Material at http://link.aps.org/supplemental/ 10.1103/PhysRevC.95.054322 for detailed information on the Hartree-Fock-Bogoliubov calculations.

[71] P. Ring and P. Schuck, The Nuclear Many-Body Problem, Texts and Monographs in Physics (Springer, Berlin, 2000).

[72] M. Bender, P.-H. Heenen, and P.-G. Reinhard, Rev. Mod. Phys. 75, 121 (2003).

[73] J. Dobaczewski, W. Satula, B. Carlsson, J. Engel, P. Olbratowski, P. Powalowski, M. Sadziak, J. Sarich, N. Schunck, A. Staszczak, M. Stoitsov, M. Zalewski, and H. Zdunczuk, Comp. Phys. Commun. 180, 2361 (2009).

[74] N. Schunck, J. Dobaczewski, J. McDonnell, W. Satula, J. Sheikh, A. Staszczak, M. Stoitsov, and P. Toivanen, Comp. Phys. Commun. 183, 166 (2012).

[75] E. Chabanat, P. Bonche, P. Haensel, J. Meyer, and R. Schaeffer, Nucl. Phys. A 635, 231 (1998).

[76] G. Fricke and K. Heilig, in Nuclear Charge Radii, LandoltBrnstein Group I Elementary Particles, Nuclei, and Atoms, Vol. 20, edited by H. Schopper (Springer, Berlin, 2004), Chap. 80, pp. 1-9.

[77] J. M. Boillos and P. Sarriguren, Phys. Rev. C 91, 034311 (2015). 
[78] T. Duguet, P. Bonche, P.-H. Heenen, and J. Meyer, Phys. Rev. C 65, 014310 (2001).

[79] V. Manea, D. Atanasov, D. Beck, K. Blaum, C. Borgmann, R. B. Cakirli, T. Eronen, S. George, F. Herfurth, A. Herlert, M. Kowalska, S. Kreim, Y. A. Litvinov, D. Lunney, D. Neidherr, M. Rosenbusch, L. Schweikhard, F. Wienholtz, R. N. Wolf, and K. Zuber, Phys. Rev. C 88, 054322 (2013).
[80] M. Bender, G. F. Bertsch, and P.-H. Heenen, Phys. Rev. C 73, 034322 (2006).

[81] J. P. Delaroche, M. Girod, J. Libert, H. Goutte, S. Hilaire, S. Péru, N. Pillet, and G. F. Bertsch, Phys. Rev. C 81, 014303 (2010).

[82] B. Bally, B. Avez, M. Bender, and P.-H. Heenen, Phys. Rev. Lett. 113, 162501 (2014). 